\title{
Mayor frecuencia de giardiosis en niños con raquitismo comparada con niños sanos de la población de Suárez, Cauca, Colombia
}

\author{
Oscar L. Sierra, Lidia N. Peñaloza, Yolanda Alarcón
}

\begin{abstract}
Resumen
Se examinaron huevos, quistes y trofozoítos de parásitos intestinales en heces de 332 niños sanos y 70 niños con raquitismo descrito como un trastorno heredado debido a malabsorción de calcio por resistencia a la vitamina $D$ en Suárez, departamento del Cauca. La frecuencia total de parasitosis fue de $65,9 \%$ y la de poliparasitismo de $10,9 \%$. Se encontraron frecuencias elevadas de giardiosis y poliparasitismo en niños con raquitismo (17,1 y 18,6\%) comparado con niños sanos $(9,0$ y $9,3 \%)$ y dichas diferencias fueron estadísticamente significativas. La vereda de La Toma, en donde se ha hallado el mayor número de raquíticos, pareció conferir un riesgo incrementado para ascariosis, pero, independiente del raquitismo. Se discuten algunos mecanismos para explicar la mayor frecuencia de parasitosis en los niños raquíticos. La incrementada frecuencia de parasitosis intestinales patógenas puede contribuir a disminuir la absorción intestinal de calcio en los niños raquíticos.
\end{abstract}

\section{Greater giardiasis frequency amongst children with rickets compared to healthy children from the town of Suárez, Cauca, Colombia}

In Suárez, Cauca, intestinal parasite ova, cysts and trophozoites were examined in faeces from 332 healthy children and from 70 children with rickets, being described as an inherited intestinal malabsorption disorder due to resistance to vitamin $\mathrm{D}$. The overall parasitosis rate was $65.9 \%$ and that for mixed parasitosis was $10.9 \%$. Increased rates of giardiosis and mixed parasitosis were found in rachitic children (17.1 and $18.6 \%$ ) as compared to healthy children $(9.0$ and $9.3 \%)$ and were statistically significant. The rural area of La Toma, where higher numbers of rachitic patients have been found, seemed to confer higher ascariosis risk, though unrelated to rickets. Some mechanisms to explain the increased parasitosis rates in rachitic children are discussed. Increased pathogenic intestinal parasitism frequency may contribute to a decrease in intestinal calcium absorption in children with rickets.

La causa principal de raquitismo es la deficiencia de vitamina D. Las ingestiones muy bajas de calcio también pueden causar raquitismo en ciertas comunidades $(1,2)$. Además, algunas formas poco comunes de raquitismo hereditario por depleción de calcio se encuentran en casi todas las poblaciones. El defecto responsable altera el metabolismo de la vitamina $\mathrm{D}$, interfiriendo con la generación del metabolito más activo la 1,25-di-hidroxivitamina 
D o su acción hormonal en tejidos blanco (3). Las enfermedades gastrointestinales y hepatobiliares que producen malabsorción y daño de la mucosa intestinal, también causan enfermedad ósea como raquitismo, osteomalacia y osteoporosis (4). Estas causas de raquitismo tienen en común el reducido transporte de calcio del compartimento intestinal hacia el compartimento sérico, ya sea por reducida disponibilidad del calcio o por transporte de calcio defectuoso por ineficiencia de los mecanismos dependientes de vitamina $D$ bajo tales condiciones.

La existencia de parásitos intestinales patógenos en comunidades con raquitismo endémico no se ha informado a pesar de un supuesto incremento en los requerimientos nutricionales de los hospederos de tales parasitosis (5). La coexistencia de parasitosis intestinales patógenas puede agregar un daño al empeorar la subyacente baja absorción intestinal de calcio. Se investigó la frecuencia de parásitos intestinales patógenos en niños con deformidades severas antes informadas, atribuibles a un raquitismo hereditario por resistencia a la vitamina $D$ y en niños sanos de las mismas veredas (6).

\section{Metodología}

Area de estudio: se estudiaron niños provenientes de las veredas de La Toma, Asnazú, Cañutico y Mindalá, del municipio de Suárez y las veredas de Palo Blanco y Honduras del municipio de Buenos Aires, ambos localizados al norte del departamento del Cauca, en el suroccidente de Colombia.

Descripción del estudio: se realizó un estudio descriptivo transversal de la frecuencia de parásitos patógenos en niños. El 17,4\% de los individuos fue clasificado como afectados de raquitismo y el $82,6 \%$ fue considerado normal; por tanto, se realizó un análisis de casos y controles no apareado.

Personas: las 402 personas participantes fueron de raza negra, de las cuales 199 eran varones $(49,5 \%)$ y 203 mujeres $(50,5 \%)$. Se procesaron muestras provenientes de 70 niños clasificados como raquíticos por tener evidentes deformidades de miembros inferiores genu valgum, genu varum o mixta. Su edad promedio fue de 10,9 6, 4 DE. Las demás 332 personas se consideraron como normales y su edad promedio fue de $10,2 \pm 5,1 \mathrm{DE}$.

Muestras fecales y procesamiento: se repartieron cajas para la recolección de muestras el día antes del análisis a los afectados de raquitismo y se repartieron al azar a los niños normales en las escuelas. Se realizaron exámenes coprológicos directos en muestras frescas con solución salina y lugol (7), y se inspeccionaron $20-40$ campos de $400 X$ en el microscopio. Se registró la presencia de huevos de las especies patógenas Ascaris lumbricoides, Trichuris trichiura y uncinaria, y la presencia de quistes o trofozoítos de Entamoeba histolytica/Entamoeba hartmanni y Giardia lamblia. El diagnóstico parasitológico fue simple ciego, pues, el diagnóstico de raquitismo lo hizo una persona que no participó en el diagnóstico parasitológico. No se realizó medición del diámetro de quistes para diferenciar entre E. histolytica y E. hartmanni; por tanto, las frecuencias de positividad se informan conjuntamente.

Análisis estadístico: se analizaron las frecuencias observadas y esperadas (8) de los parásitos patógenos en la población en tablas de $2 \times 2$ en Epilnfo 6.01 (afectados/sano vs. positivo/negativo) y se calcularon las razones de productos cruzados (odds ratio, OR) (8) entre afectados y sanos para cada parásito. Ninguna frecuencia fue menor de 5 para las comparaciones de categorías generales. Las diferencias se calcularon por chi cuadrado de Pearson (8). Un P de 0,05 o menor indicó significancia estadística.

\section{Resultados}

Las frecuencias de las parasitosis patógenas y no patógenas aparecen en el cuadro 1 , relacionadas según las veredas estudiadas y el número de raquíticos positivos para cada parasitosis. $65,9 \%$ de todos los individuos 
Cuadro 1. Frecuencia de parasitismo intestinal en la población estudiada y en raquíticos. Distribución por veredas de los municipios de Suárez y Buenos Aires, Cauca.

\begin{tabular}{|c|c|c|c|c|c|c|c|c|c|c|c|}
\hline Parásito & La Toma & 1a \% & \multicolumn{2}{|c|}{$\begin{array}{c}\text { Suárez } \\
\text { Asnazú }{ }^{1} \%\end{array}$} & \multicolumn{2}{|c|}{ Mindalá \% } & $\begin{array}{l}\text { Buenos } \\
\text { Palo Blanco }\end{array}$ & \multirow[t]{2}{*}{$\begin{array}{l}\text { Aires } \\
\%\end{array}$} & \multirow[t]{2}{*}{ Total } & $\begin{array}{l}\text { Raquíticos } \\
\text { Positivos }\end{array}$ & s $\%$ \\
\hline Patógenos & & & & & & & & & & & \\
\hline A. lumbricoides & 32 & 18,4 & 16 & 9,1 & 4 & 18,2 & 8 & 26,7 & 60 & 13 & 18,6 \\
\hline G. lamblia & 11 & 6,3 & 21 & 11,9 & 5 & 22,7 & 5 & 16,7 & 42 & 12 & 17,1 \\
\hline $\begin{array}{l}\text { E. histolytica/E. hartmanni } \\
\text { No patógenos }\end{array}$ & 88 & 50,6 & 88 & 50 & 14 & 63,6 & 12 & 40 & 202 & 34 & 48,6 \\
\hline E. nana & 4 & 2,3 & 5 & 2,8 & 0 & 0 & 0 & 0 & 9 & 2 & 2,9 \\
\hline E. coli & 7 & 4 & 7 & 4,0 & 3 & 13,6 & 6 & 20 & 23 & 7 & 10 \\
\hline I. butchslii & 18 & 10,3 & 7 & 4,0 & 3 & 13,6 & 1 & 3,3 & 29 & 5 & 7,1 \\
\hline Poliparasitismo ${ }^{3}$ & 21 & 12,1 & 10 & 5,7 & 6 & 27,3 & 5 & 16,7 & $44^{4}$ & 13 & 18,6 \\
\hline Raquíticos & 30 & 17,2 & 12 & 6,8 & 11 & 50 & 17 & 56,7 & 70 & 70 & 100 \\
\hline Total vereda & 1741 & 100 & 176 & 100 & 22 & 100 & 30 & 100 & 402 & - & - \\
\hline
\end{tabular}

1 Incluye a la vereda de Cañutico

${ }^{2}$ Incluye a la vereda de Honduras

${ }^{3}$ Dos o más parásitos patógenos

${ }^{4}$ Incluye dos poliparasitismos, uno con uncinaria y otro con tricocéfalo

estudiados presentó positividad para parásitos patógenos. La frecuencia de poliparasitosis total, definida por tener dos o más parásitos patógenos (mixta), fue de 10,9\% (figura 1). Hubo mayor frecuencia de parasitosis en afectados que en sanos ( $A$. lumbricoides, $G$. lamblia, $E$. coli), de las cuales sólo $G$. lamblia tuvo una asociación estadística $(p=0,0438)$ con raquitismo y la frecuencia de afección por $G$.

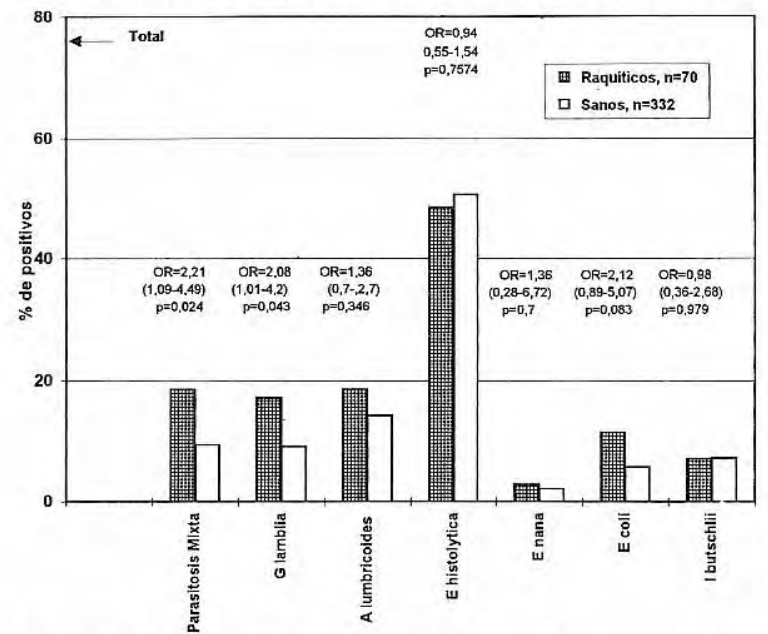

Figura 1. Porcentaje de positividad de parásitos intestinales en niños con raquitismo y sanos. Comparación de riesgo relativo indirecto (Odds ratio, $O R$ ) entre raquíticos y sanos para cada parasitosis e intervalo de confianza de $95 \%$. Poliparasitismo: parasitosis mixta. Entre paréntesis aparece el valor de $\mathrm{P}$ calculado mediante chicuadrado de Pearson en tablas de $2 \times 2$. lamblia fue de $17,1 \%$ en raquíticos frente a $9,0 \%$ en sanos. EI OR o riesgo relativo indirecto es de 2,08 [IC95=1,01-4,3]. La prevalencia de infección por $E$. histolytica/E. hartmanni fue cercana a $50 \%$ en ambos grupos. Se encontraron dos casos de uncinaria, un caso de tricocefalosis y no se detectó teniosis ni estrongiloidiosis. El poliparasitismo se encontró asociado a raquitismo con un riesgo relativo indirecto de 2,21 [IC95=1,09-4,49] y mayor frecuencia en raquíticos $(18,6 \%)$ frente a sanos $(9,3 \%)$ cuya diferencia fue estadísticamente significativa $(p=0,0245)$. En la vereda de La Toma, se encontraron 30 raquíticos de 174 sujetos analizados $(17,2 \%)$ y en Asnazú 13 raquíticos de 176 analizados $(7,3 \%)$, diferencia que fue significativa $(p=0,0027)$ en la muestra analizada.

La mayoría de los raquíticos $(82,9 \%)$ en este estudio viven en áreas rurales sin alcantarillado y $\sin$ acueducto (La Toma, Mindalá y Palo Blanco). Se examinó si existía una asociación de las parasitosis entre las veredas de La Toma y Asnazú/Cañutico considerando que se analizó un número total similar de sujetos y por tener Asnazú/Cañutico menor frecuencia de raquíticos. En el cuadro 2 se observa que sólo se encontró positividad general y significancia estadística para ascariosis, siendo mayor en La Toma aunque no asociado al raquitismo. 
Se realizó la comparación de las frecuencias de los parásitos patógenos por tres grupos de edad y por sexo en raquíticos y sanos, la cual aparece en el cuadro 3 . Se observa que no hubo asociación de parasitosis patógenas por edad o sexo, excepto ciertas comparaciones que dieron significativas. En tales casos, el número de individuos en alguna de las casillas de la tabla de $2 \times 2$ es menor de 5 sujetos y, por tanto, la asociación emergente se considera carente de validez. En poliparasitismo, aparece asociación estadística entre raquíticos y sanos para dos grupos de edad lo cual confirma lo encontrado en el grupo completo.

El porcentaje de raquíticos en esta muestra fue de $17,4 \%$, lo cual se explica por el diseño de casos y controles no apareados aunque equilibrados por edad, sexo y raza.

\section{Discusión}

La prevalencia del raquitismo en Suárez fue informada como de $2,2 \%$ según Iglesias et al. (9) en 1992 y de 5,1\% según Bermúdez et al. (10) por encuesta de prevalencia en 1996. La prevalencia que aquí informamos, basados en el conocimiento de cada uno de los casos y dividido por la población total de Suárez según censo nacional de 1993 , es de $0,93 \%$.

El examen microscópico de las heces es un método conveniente para estudios epidemiológicos por su simplicidad y bajo costo. Los métodos invasores como los aspirados duodenales, la biopsia intestinal o el drenaje biliar (11) se han considerado más sensibles para giardiosis. Sin embargo, un estudio de Duque y Nicholls en 1997 en el Instituto Nacional de Salud en Bogotá, demostró que el examen coprológico es más sensible, al compararlo con los métodos invasores (12). El examen seriado de heces aumenta la detección y se recomienda cuando la sospecha clínica de giardiosis es considerable. Sin embargo, para estudios epidemiológicos el criterio difiere, pues, algunos autores (13-15) han demostrado que, en la primera muestra de heces, se hace el $90 \%$ de los diagnósticos, especialmente si se utilizan métodos de concentración y fijación.
Como se utilizó el examen coprológico directo, la prevalencia real de giardiosis podría ser más alta en la población examinada. Es de esperarse que la detección de parásitos en heces refleje proporcionalmente las diferencias de infección entre los individuos o de aquellos más severamente infestados.

Se cree que los parásitos patógenos intestinales afectan el estado nutricional y generan patología intestinal absortiva, obstructiva, alérgica y de motilidad (16). Sin embargo, pocos parásitos tienen esta asociación bien establecida, pues existen factores de confusión relacionados con su alta prevalencia en poblaciones con desnutrición, problemas sanitarios y pobreza generalizada. No se conocen bien los mecanismos por los cuales los parásitos patógenos alteran el estado nutricional. Dentro de los mejor descritos están las uncinarias que ulceran la mucosa intestinal y succionan sangre causando anemia y pérdida de proteínas. Otro parásito es $G$. lamblia que se fija a la superficie intestinal por medio del disco de succión lo cual provoca una respuesta inflamatoria y atrofia de las vellosidades que disminuye la superficie de absorción. G. lamblia es un parásito flagelado con capacidad de infectar las vías biliares; se ha descrito la invasión activa de la mucosa intestinal (17). Asociado a la giardiosis, se ha encontrado sobrecrecimiento bacteriano de las porciones altas del intestino y aclorhidria estomacal con elevado $\mathrm{pH}$ gástrico que perturba la acidez como mecanismo de defensa contra gérmenes. La giardiosis ocasiona esteatorrea, malabsorción de carbohidratos, vitaminas A y B12 e intolerancia a la lactosa (18). Se sabe que la vitamina D tiene circulación enterohepática (19) y si hay pérdida de sales biliares por esteatorrea podría ocasionar déficit de vitamina $D(20)$. Se ha descrito que los ácidos grasos no esterificados capturan el calcio (21) y que serían arrastrados por esteatorrea generando malabsorción de calcio. La aclorhidria es un factor que limita la absorción de calcio ya que el ácido estomacal promueve la disolución de las sales de calcio y evita su precipitación (22). La 
diarrea osmótica por malabsorción de lactosa puede reducir la absorción de calcio o la intolerancia a la lactosa puede desestimular el consumo de lácteos con alto contenido de calcio. Se ha encontrado asociación entre la intolerancia a la lactosa y la osteoporosis (23).

Sobre estas bases, G. lamblia ha sido un parásito asociado a la malabsorción de nutrientes. Creemos que la coexistencia de giardiosis y parasitismo intestinal en un subgrupo de niños con raquitismo puede empeorar el transporte intestinal de nutrientes claves para el desarrollo óseo, de por sí ya disminuido por su enfermedad.

Desconocemos la razón de la mayor frecuencia de giardiosis y poliparasitismo en niños con raquitismo comparados con niños sanos de la misma región. La resistencia a la vitamina $D$, que es la causa propuesta para esta enfermedad en la población estudiada, ha sido asociada a inmunodeficiencia (24) que podría predisponer a una mayor frecuencia de giardiosis. En la hipogammaglobulinemia (25), se presenta una prevalencia incrementada de giardiosis. Es interesante que el raquitismo nutricional haya sido asociado con mayor morbilidad por neumonía en niños del Africa (26). La asociación estadística encontrada no implica una relación causal, pero los casos informados en la literatura sugieren mecanismos relacionados con nuestros pacientes.

La ausencia de asociación en la positividad general por veredas parece no relacionarse con las condiciones sanitarias de la vereda a excepción de la ascariosis y del poliparasitismo, en virtud de que la frecuencia de ascariosis influencia la de poliparasitismo. La Toma es un asentamiento típicamente rural mientras Asnazú tiene un aspecto más urbano.

Los niños con patologías intestinales por malabsorción de nutrientes y parasitismo intestinal sobreagregado pueden beneficiarse de la terapia antiparasitaria. Creemos interesante explorar mejor la asociación de estos dos trastornos con la ayuda de métodos serológicos para la detección de la respuesta a G. lamblia.

\section{Agradecimientos}

Este estudio fue financiado por COLCIENCIASBID proyecto \#2104-04-018-96 y el Instituto Nacional de Salud. Agradecemos a las promotoras de salud de Suárez, a la doctora Blanca Chávez, directora del Centro Hospital de Suárez, al licenciado Hernando Ramírez, alcalde de Suárez y a la Empresa de Energía del Pacífico EPSA SA, ESP. Agradecemos a las personas que aportaron sugerencias a este manuscrito.

\section{Referencias}

1. Pettifor JM, Ross P, Wang J, Moodley G, Couper-Smith J. Rickets in children of rural origin in South Africa: is low dietary calcium a factor? J Pediatr 1978;92:320-4.

2. Okonofua F, Gill DS, Alabi ZO, Thomas M, Bell JL, Dandona P. Rickets in Nigerian children: a consequence of calcium malnutrition. Metabolism 1991;40:209-13.

3. Marx SJ, Liberman UA, Eil C, Gamblin GT, DeGrange DA, Balsan S. Hereditary resistance to 1,25-dihydrovitamin D. Recent Prog Horm Res 1984;40:589-620.

4. Sitrin M, Meredith S, Rosenberg IH. Vitamin D deficiency and bone disease in gastrointestinal disorders. Arch Intern Med 1978;138:886-8.

5. Howes Calloway D. Nutritional requirements in parasitic diseases. Rev Infec Dis 1982;4:891-5.

6. Giraldo A, Pino W, García-Ramírez LF, Pineda M, Iglesias A. Vitamin D dependent rickets type II and normal vitamin $D$ receptor cDNA sequence. A cluster in a rural area of Cauca, Colombia with more than 200 affected children. Clin Genet 1995; 48:57-65.

7. Botero D, Restrepo M. Técnicas de laboratorio en parasitología médica. En: Parasitosis humanas. Segunda edición. Medellín: Corporación para las Investigaciones Biológicas, CIB; 1982:379-403.

8. Remington RD, Schork MA. Frequency data. In: Statistics with applications to the biological and health sciences. Second Edition. New Jersey: Prentice Hall; 1985;205-34.

9. Iglesias-Gamarra A, Vásquez-Lamadrid J, AbudMendoza C. Los errores innatos del metabolismo causantes de enfermedad ósea. En: Enfermedades metabólicas del hueso. Bogotá: Instituto Nacional de Salud; $1992 . p .158$. 
10. Bermúdez A, Acosta A, Chavez B, Sánchez C. Encuesta de prevalencia de enfermedad que cursa con deformidad en las piernas, en la población de Suárez, departamento del Cauca, julio de 1996. Bogotá: Ministerio de Salud, Instituto Nacional de Salud. Informe1.3, Servicio de Epidemiología Aplicada, Trabajos del Entrenado, 1997.

11. Sotto Escobar A. Giardiosis. La Habana: Editorial Científico-Técnica; 1987.p.96

12. Duque S, Nicholls RS. Consideraciones sobre el diagnóstico de la giardiasis. Biomédica 1997; 17(Supl.2):106-9.

13. Senay H, MacPherson D. Parasitology: diagnostic yield of stool examination. Can Med Assoc J 1989; 140:1329-31

14. Montessori GA, Bischoff L. Searching for parasites in stool: once is ususally enough. Can Med Assoc J 1987;137:702.

15. Giorkos TW, MacLean JD, Law CG. Absence of significant differences in intestinal parasite prevalence estimates after examination of either one or two stool specimens. Am J Epidemiol 1989; 130:976-80.

16. Rosenberg IH, Bowman BB. Intestinal physiology and parasitic diseases. Rev Infect Dis 1982; 4:763-7.

17. Stevens DP. Giardiosis: host-pathogen biology. Rev Infect Dis 1982;4:851-8.

18. Solomons NW. Giardiosis: nutritional implications. Rev Infect Dis 1982;4:859-69.
19. Arnaud SA, Goldsmith RS, Lambert PW, Go VLW. 25-hydroxyvitamin D3: evidence for enterohepatic circulation in man. Proc Soc Exp Biol Med 1975;149:570-2.

20. Bikle DD. Osteoporosis in gastrointestinal, pancreatic and hepatic diseases. In: Marcus R, Feldman D, Kelsey J, editors. Osteoporosis. San Diego, CA: Academic Press; 1996.pp.863-4.

21. Birge SJ, Avioli LV. Pathophysiology of calcium and phosphate absorptive disorders. In: Avioli LV, Krane $\mathrm{SM}$, editors. Metabolic bone disease and clinically related disorders. WB Saunders Co.; 1990.pp.196221.

22. Levenson DI, Bockman RS. A review of calcium preparations. Nutr Rev 1994;52:221-32.

23. Birge SJ, Keutmann HT, Cuatrecasas P, Whedon GD. Osteoporosis, intestinal lactase deficiency and low dietary calcium intake. $N$ Engl J Med 1967;276:445-8.

24. Etzioni A, Hochberg Z, Pollak S, Meshulam T, Zakut V, Tzehoval E, Keisari Y, Aviram Y, Spirer Z, Benderly A, Weisman Y. Defective leukocyte fungicidal activity in end-organ resistance to 1,25dihydroxyvitamin D. Pediatr Res 1989;25:276-9.

25. Ament ME, Rubin CE. Relation of giardiosis to abnormal intestinal structure and function in gastrointestinal immunodeficiency syndromes. Gastroenterology 1972;62:216-26.

26. Muhe L, Lulseged S, Mason KE, EAF Simoes. Case-control study of the role of nutritional rickets in the risk of developing pneumonia in Ethiopian children. Lancet 1997;349:1801-4. 